\title{
PRESTRESS FOR MAXIMUM STRENGTH
}

\author{
ZENON MROZ \\ Institute of Basic Technical Problems, Polish Academy of Sciences, Warsaw
}

and

J. E. TAYLOR

The University of Michigan, Ann Arbor, Michigan

\begin{abstract}
The problem of designing prestress to maximize elastic capacity is treated analytically. Our formulation models structures comprised of a one-material elastic continuum, subject to a single deterministic load configuration.

The equations needed to predict the optimal prestress design are derived. They are shown to comprise necessary and sufficient conditions for global maximum strength in the unconstrained design problem. The theory is demonstrated on the design of prestress for a thick-walled cylinder.
\end{abstract}

\section{INTRODUCTION}

FREQUENTLY the structural function of a structure or machine part might be enhanced through the installation of an appropriate initial stress state. Indeed, prestress is primary to structural integrity for some material composites, such as prestressed concrete. Otherwise, for a structure made of either a single material or a composite, structural response is almost always sensitive to initial stress state.

At least theoretically, the option to use prestress in design exists in proportion to this sensitivity. Yet among topics in structural mechanics, there appears to be relatively little literature on basics in the design of prestress. Some of the literature on particular design problems is listed in [1] and [2]. An unusual application of structural prestress is treated in [3], while [4] presents a method to evaluate optimal prestress. A formulation is given in [5] for problems in the design of prestress to extremize the Euler load. Optimal prestress relative to plastic collapse and relative to elastic capacity are related through theorems stated recently by Nagtegaal [6].

This paper provides an analytical formulation for problems arising in the design of prestress to maximize carrying capacity. The results apply to structures made of a single material, where the structure experiences just one (deterministic) loading environment. The development leading to these results is otherwise general; it is written in the next section for an elastic continuum but the argument is similar for any structural element or system where prestress might be used to increase elastic capacity. It also proves possible to predict the collapse load for von Mises yield condition, simply by interpreting in an appropriate way the outcome of our investigation for elastic structures.

An example is given to indicate how the theory of this paper may be used to predict an optimal prestress configuration. However, no attempt is made to otherwise consider the technical situations where prestress might be employed to practical advantage. Nor 
do we consider the technical processes by which a desired prestress state might be installed in a structure.

\section{FORMULATION}

Our first objective is to show that the particular prestress field which maximizes the total strain energy of the loaded system, within a prescribed limit on the local capacity of the material, is the optimal one. Here optimum is identified with maximum load carrying capacity. The local material capacity is stated in terms of a bound on permissible values of the specific strain energy. This part of the demonstration of optimum prestress makes use of the Virtual Work equation and Betti's reciprocity relation. Our development is intended to apply to a linearly elastic solid, although so far as dependence on these fundamental relations is concerned a more general interpretation is possible (see Fung [7]).

The total stress $\boldsymbol{\sigma}$ is represented as the simple sum of the initial or prestress field $\boldsymbol{\sigma}^{I}$ and the stress $\sigma^{L}$ associated with loading:

$$
\sigma=\sigma^{I}+\sigma^{L} .
$$

We take $\sigma^{I}$ to symbolize any prestress field among the set of self-equilibrated fields with equal strain energy. In other words, it is required that the prestress strain energy $U_{I}\left(\sigma^{I}\right)$ satisfy:

$$
U_{I}\left(\sigma^{I}\right)=A^{2}, \text { constant }
$$

The value of the constant $A$ is to be determined later.

For stress-strain and displacement fields $\sigma^{L}, \varepsilon^{L}$ and $\mathbf{u}^{L}$ associated with tractions $T$ along portion $S_{T}$ of the boundary, the virtual work equation states:

$$
\int_{S_{T}} T_{u^{L}} \mathrm{~d} S=\int_{R} \sigma^{L} \varepsilon^{L} \mathrm{~d} V
$$

Region $R$ corresponds to the region occupicd by structural material. Also, from Betti's theorem we may write

$$
\int_{R} \sigma^{L} \varepsilon^{L} \mathrm{~d} V=\int_{R} \sigma^{L} \varepsilon^{L} \mathrm{~d} V=0 .
$$

The last equality in equation (4) follows from the fact that the $\sigma^{I}$ is self-equilibrated. $\sigma^{I}$ represents any admissible prestress field, that is to say, any self-equilibrated field that meets equation (2).

Making use of equations (1) and (4), equation (3) is rewritten:

$$
\left.\int_{S_{T}} \mathbf{T}^{L} \mathbf{u}^{L} \mathrm{~d} S=\int_{R}\left[\boldsymbol{\sigma}^{L}+\sigma^{I}\right)\left(\varepsilon^{L}+\varepsilon^{I}\right)-\sigma^{I} \varepsilon^{I}\right] \mathrm{d} V \equiv \int_{R}\left(\sigma \varepsilon-\sigma^{I} \varepsilon^{I}\right) \mathrm{d} V .
$$

Equation (5) is general. If superscript * is used to identify the optimal solution, we may write in particular

$$
\int_{S_{T}} \mathbf{T}^{L} \mathbf{u}^{L *} \mathrm{~d} S=\int_{R}\left[\left(\sigma^{L}+\sigma^{I *}\right)\left(\varepsilon^{L}+\varepsilon^{I *}\right)-\sigma^{I *} \varepsilon^{I *}\right] \mathrm{d} V \equiv \int_{R}\left(\sigma^{*} \varepsilon^{*}-\sigma^{I *} \varepsilon^{I *}\right) \mathrm{d} V
$$


We write a similar equation for the situation of arbitrary admissible prestress $\boldsymbol{\sigma}^{I}$. The load in this case is designated $\lambda \mathbf{T}^{L}$, and the associated loading stresses are $\lambda \sigma^{L}$.

$$
\lambda \int_{S_{T}} \mathrm{~T}^{L} \mathbf{u}^{L *} \mathrm{~d} S=\int_{R}\left[\left(\lambda \sigma^{L}+\sigma^{I}\right)\left(\varepsilon^{L}+\varepsilon^{I *}\right)-\sigma^{I} \varepsilon^{I *}\right] \mathrm{d} V \equiv \int_{R}\left(\sigma \varepsilon^{*}-\sigma^{I} \varepsilon^{I *}\right) \mathrm{d} V .
$$

As implied by the last equality of equation $(5 b)$

$$
\boldsymbol{\sigma}=\lambda \sigma^{L}+\boldsymbol{\sigma}^{I}
$$

Equation (5a) is subtracted from equation (5b) and use is made of equation (2) to obtain:

$$
(\lambda-1) \int_{S_{T}} \mathbf{T u}^{L *} \mathrm{~d} S=-\int_{R}\left(\sigma^{*}-\sigma\right) \varepsilon \mathrm{d} V+U\left(\sigma^{I *}-\sigma^{I}\right) .
$$

Using the definitions of $\sigma$ and $\sigma^{*}$, along with equation (3), equation (6) may be reduced to

$$
(\lambda-1) \int_{S_{T}} \mathbf{T} \mathbf{u}^{L *} \mathrm{~d} S=-(1-\lambda)^{2} U\left(\sigma^{L *}\right)-2 U\left(\sigma^{*}\right)+2 U(\sigma) .
$$

Therefore since $U\left(\sigma^{*}\right), U(\sigma)$ and $U\left(\sigma^{L *}\right)$ are positive definite for nonzero load, we find $\lambda<1$ if $U(\sigma)<U\left(\sigma^{*}\right)$.

In other words, it has been shown that the prestress field which maximizes total strain energy maximizes the load capacity. We have now to indicate how this maximizing prestress field might be determined. Recall that the total stress field is not to violate a specified upper bound constraint. If the strain energy is expressed

then this constraint takes the form

$$
U(\sigma)=\int_{R} E(\sigma) \mathrm{d} V
$$

$$
E(\boldsymbol{\sigma}) \leq \widetilde{E},
$$

where $E$ is simply specific strain energy and $\tilde{E}>0$ represents the specified bound. We note that $E$ is proportional to the square of the octahedral shear stress.

One might at this stage seek to establish general necessary conditions for the extrema of $U(\sigma)$ within the constraint equations (8) and (2). However, this procedure requires that several auxiliary variables be introduced and is unnecessarily lengthy. We will instead simply state the (not unfamiliar) necessary condition that pertains, and verify that it is sufficient to guarantee a maximum of $U\left(\sigma^{*}\right)$. The cited necessary condition is

$$
E\left(\sigma^{*}\right)=\widetilde{E}, \text { constant }
$$

In other words, if the constraint (8) is interpreted using slack function $s^{2}$ in the form

$$
E-E(\sigma)-s^{2}=0,
$$

according to equation (9), $s \equiv 0$ for the optimum solution. This is easily verified. Substituting for $E(\sigma)$ from equation (10) into $U(\sigma)$ of equation (7), we obtain

$$
\begin{aligned}
U(\boldsymbol{\sigma}) & =\int_{R} E(\boldsymbol{\sigma}) \mathrm{d} V \\
& =\int_{R} \tilde{E} \mathrm{~d} V-\int_{R} s^{2} \mathrm{~d} V,
\end{aligned}
$$


but according to equation (9), $\int \tilde{E} \mathrm{~d} V=\int E\left(\sigma^{*}\right) \mathrm{d} V=U\left(\sigma^{*}\right)$. Thus (11) becomes

$$
U(\sigma)=U\left(\sigma^{*}\right)-\int_{R} s^{2} \mathrm{~d} V
$$

whereby we have $U\left(\sigma^{*}\right)>U(\sigma)$ for $\sigma^{*} \neq \sigma$.

We note that an interpretation may now be given to the value of the constant $C^{2}$ in equation (2). That is, for a given load the prestress energy should take on a value appropriate to the condition equation (9). Note that with the constraint on prestress expressed as in equation (2), there is no implication on the local values of the prestress from the condition just described.

\section{AN EXAMPLE}

Given the existence of an optimum prestress field $\sigma^{I *}$ for a particular structure and load $\mathbf{T}^{L}$, we have shown equation (9) to be necessary and sufficient for the determination of the $\sigma^{I *}$. We apply these results to obtain the optimum solution in the Lamé problem, as an example application.

The problem is written most conveniently in terms of a stress function, say $\phi$. In the axisymmetric situation, equilibrium is satisfied if :

$$
\begin{aligned}
\sigma_{r r} & =\phi / r \\
\sigma_{\theta \theta} & =\phi^{\prime} \\
\sigma_{r \theta} & =0
\end{aligned}
$$

where prime indicates derivative w.r.t.r. Suppose the cylinder extends from $r=a$ to $r=b$, and is subject to internal pressure $p$ only. Then we seek a function $\phi(r)$ defined over $r \in[a, b]$, with values $\phi(a)=-a p$ at $r=a$ and $\phi(b)=0$ at $r=b$. The optimum solution, say $\phi^{*}$, must also satisfy equation (9). For this latter condition, we note that $E(r) \sim\left[(\phi / r)^{2}+\right.$ $\left.\phi^{\prime 2}-2 v \phi \phi^{\prime} / r\right]$, and so the requirement equation (9) is expressed :

$$
\left(\phi^{*} / r\right)^{2}+\left(\phi^{*^{\prime}}\right)^{2}-2 v \phi^{*} \phi^{*^{\prime}} / r=\tilde{E}
$$

Equation (13) is satisfied by a function $\phi \sim r$. It is not possible to meet the boundary conditions with this solution, however, so another solution must be found by integrating equation (13). To facilitate this step, the equation is transformed according to:

$$
\begin{aligned}
\phi & =C r \psi, \quad C=\text { constant } \\
1 \leq \rho & =r / a \leq \rho_{0} .
\end{aligned}
$$

The results of this transformation are:

$$
\psi^{\prime 2}+e \psi^{\prime} \psi / \rho+e\left(\psi^{2}-1\right) / \rho^{2}=0
$$

with constants

$$
\begin{aligned}
e & =2(1-v) \\
v & =\text { Poisson ratio } \\
C^{2} & =\widetilde{E} / e
\end{aligned}
$$


and

$$
\begin{aligned}
& \psi(1)=-\rho / C \\
& \psi(0)=0
\end{aligned}
$$

Equation (15) is in separable form, i.e.

$$
\int \mathrm{d} \psi /\left\{-e^{\frac{1}{2}} \psi \pm\left[4-(4-e) \psi^{2}\right]^{\frac{1}{2}}\right\}=\left(e^{\frac{1}{2}} / 2\right) \operatorname{Ln} \rho+B
$$

The result from integration of the L.S. of equation (17) is expressed in terms of the function $\beta(\rho)$ defined by

This result is :

$$
2 \cos \beta(\rho)=-\psi(\rho)
$$

$$
\mp \beta-e^{\frac{1}{2}} \operatorname{Ln}\left(e^{\frac{1}{2}} \cos \beta \mp \sin \beta\right)=(1+e)\left(e^{\frac{1}{2}} / 2\right) \operatorname{Ln} \rho+B .
$$

Equation (16) requires

$$
\begin{aligned}
\cos \beta(1) & =p / 2 C \\
\cos \beta\left(\rho_{o}\right) & =0 .
\end{aligned}
$$

If the positive signs are chosen in equation (19), the upper limit on $\beta(\rho)$ is $\beta\left(\rho_{0}\right)=\pi / 2$. To complete a specific example, say the load $p$ relates to the (specified) material capacity according to $(p / 2 C)=0.25$. Then $\beta=\cos ^{-1} 0.25=1.32$, and from equation (19) we find $B=0.453$. Applying equation (19) to the boundary $\rho=\rho_{o}$ yields $\rho_{o}=1.46$, and the solution is completely determined. The radial stress as a function of $\rho$ for this case is indicated in Fig. 1. The prestress field say $\phi^{I}$ is now obtained from

$$
\phi^{1}=\phi-\phi^{L}
$$

where $\phi^{L}$ represents the (known) Lamé Solution.

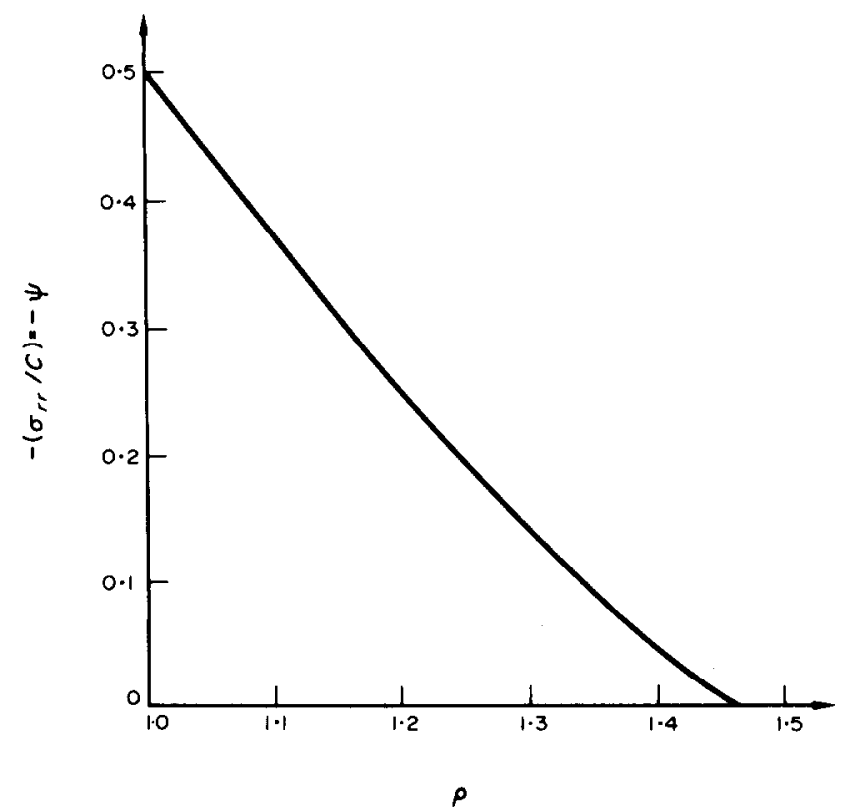

FIG. 1. 
All solutions for optimum prestress of the internally loaded cylinder may be summarized on a single plot of $\rho_{o}$ vs $p / 2 C$ (see Fig. 2). The parameter $p / 2 C$ is the so called design index. The upper limit point on this plot corresponds to the situation where any increase in internal pressure would result in a violation of the limit of material capacity $E(\rho) \leq \tilde{E}$, regardless of prestress. In other words, there is no occasion to build such a cylinder thicker than the one indicated by this point.

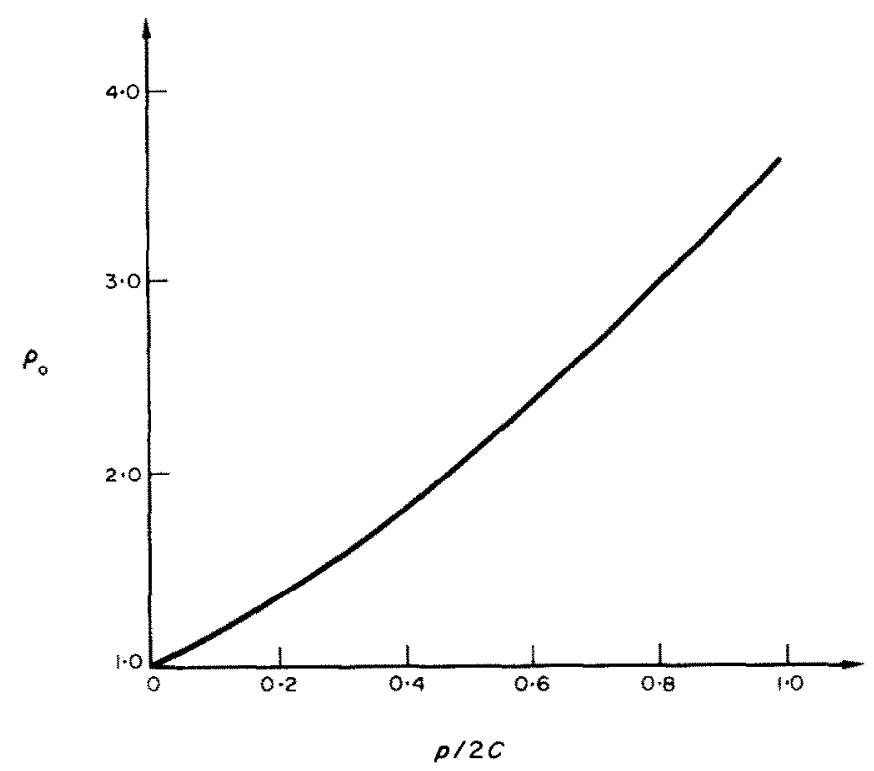

FIG. 2.

\section{DISCUSSION}

In the present paper, we assumed the form of the structure as given and considered only variation of the prestress field. It was demonstrated how to predict the optimum prestress field from among arbitrary initial stress states. Even where the choice among prestress states is not free, the conclusion drawn from equation (6a) still applies. That is, considering constrained prestress states the load capacity is maximized by the particular prestress field which maximizes total strain energy.

Furthermore, the results presented here do not depend on homogencity of material, and the theorem therefore pertains to situations where prestress is introduced through prestressing cables, fibers, or by the installation of shrinkage or misfit stresses. Thus, the result, equation (6a), may be useful as a guide to the selection of prestress in cases where dissimilar materials are combined with prestressing to meet certain strength or other technological constraints.

Also, as noted in the Introduction, the collapse load (for ideally plastic behavior) may be predicted directly from a solution of the unconstrained prestress design problem. To see this, note that if the Poisson ratio is set equal to $\frac{1}{2}$ in equation (13), and the right side is taken equal to the square of the yield stress, the equation simply expresses the 
von Mises yield condition. Thus, the optimal prestress solution in this case corresponds to uniform satisfaction of the yield condition, and the solution therefore predicts the collapse load. We observe as well that with our simple one-parameter load, this collapse capacity equals the shakedown load for the structure.

Acknowledgment-The work reported in this paper received support in part (J. E. Taylor) under National Science Foundation Grant GK-31476.

\section{REFERENCES}

[1] Z. WASIUTYNSKI and A. BRANDT, The present state of knowledge in the field of optimum design of structures. Appl. Mech. Rev. 16 (5) (May 1963).

[2] M. I. Reitman, Optimal design of structures-References. Ministry of Architecture, U.S.S.R., Moscow (1969).

[3] STEFAN Jendo, Optimum design of axially-symmetric suspended structures. Roumanian J. Tech. Sci. Appl. Mech. 16 (3) (1971).

[4] G. RozvanY, Optimum synthesis of prestressed structures. Proc. ASCE 90 (ST-6) 189-211 (1964).

[5] J. E. TAYLOR, Optimal prestress against buckling-An energy approach. Int. J. Solids Struct. 7, 213-223 (1971).

[6] J. C. Nagtegaal, On optimal design of prestressed elastic structures. Int. J. Mech. Sci. 14, 779-781 (1972).

[7] Y. C. Fung, Foundations of Solid Mechanics. Prentice-Hall, New Jersey (1965).

(Received 20 October 1972; revised 16 April 1973)

Абстракт-Обрабатывается аналитически задача расчета предварительного напряжения, с целью увеличения до крайности упругой способности. Конструкцни для предложенных моделей изготовлены из одло-материальной, упругой сплошной среды, подчиненной единичной, детерминисгической конфигурации нагрузки.

Определяются уравнения, нужны для предсказания оптимального расчета предварительного напраяжения. Доказывается, что зти уравнения заключают в себе необходимые и достатоуные условыя, для рассматривания в целом максимальной прочности, в задаче расчета конструкции, не стесненной связами. Теория указана на примере расчета предварительного напряжения толстостенного цилинлра. 